\title{
Analysis of heat capacity and Mössbauer data for $\mathrm{LuZnSn}_{2}$ compound
}

\author{
Kazimierz Łątka, \\ Janusz Przewoźnik, \\ Jan Zukrowski, \\ Yuriy Verbovytskyy, \\ Antonio Pereira Gonçalves
}

\begin{abstract}
New analysis of heat capacity data is presented for $\mathrm{LuZnSn}_{2}$ compound that takes into account anharmonic effects together with the existence of Einstein modes. ${ }^{119 m} \mathrm{Sn}$ Mössbauer spectroscopy was used to monitor the hyperfine parameters at the two crystallographically inequivalent Sn sites in the studied compound. The problem of non-unique mathematical resonance spectrum description and the problem how to choose physically meaningful set of hyperfine parameters will be thoroughly discussed. Measured quadrupole interaction constants by ${ }^{119 \mathrm{~m}} \mathrm{Sn}$ Mössbauer spectroscopy give estimations for $V_{z z}$ component of electric field gradient tensor at both $\mathrm{Sn}$ sites in $\mathrm{LuZnSn}_{2}$.
\end{abstract}

Key words: heat capacity • Mössbauer studies • rare earth ternary compounds

K. Łątka

M. Smoluchowski Institute of Physics,

Jagiellonian University,

11 Prof. S. Łojasiewicza Str., 30-348 Kraków, Poland,

Tel.: +48 12663 5668, Fax: +48 12633 7086,

E-mail: uflatka@cyf-kr.edu.pl

\section{Introduction}

Recently, new ternary intermetallic compounds $\mathrm{RZnSn}_{2}(\mathrm{R}=\mathrm{Y}, \mathrm{Gd}, \mathrm{Tb}, \mathrm{Dy}, \mathrm{Ho}, \mathrm{Er}, \mathrm{Tm}, \mathrm{Lu})$ were synthesized and it was shown that they crystallize in the tetragonal $\mathrm{HfCuSi}_{2}$ structure type [1]. These compounds belong to the rich family of $\mathrm{R}(\mathrm{A})-\mathrm{T}-\mathrm{X}$ $(\mathrm{R}-$ rare earth or $\mathrm{A}-$ actinide element, $\mathrm{T}-d$ metal, $\mathrm{X}-p$ element) ternary intermetallics [2-21], which present a variety of crystal and magnetic structures and a plethora of interesting physical properties, including complex magnetism, unconventional superconductivity, and intermediate valence. Magnetic susceptibility and heat capacity results show that $\mathrm{LuZnSn}_{2}$ compound does not undergo any magnetic phase transition down to $1.9 \mathrm{~K}[1,22]$, and therefore this compound is an ideal reference material for the estimation of magnetic contributions to heat capacity of magnetically ordered isostructural compounds.

The aim of this work is to present and discuss a new analysis of heat capacity data [22] obtained for $\mathrm{LuZnSn}_{2}$ with the help of an extended Debye model that fits experimental data in whole range of temperatures in contrast to the results obtained earlier [22] where simple Debye model was used. Additionally, the results of preliminary Mössbauer investigation made with ${ }^{119} \mathrm{Sn}$ source will be reported and discussed for the compound under study.
Received: 18 June 2014

Accepted: 2 November 2014

\author{
Campus Tecnológico e Nuclear, \\ Universidade de Lisboa, \\ Estrada Nacional 10, 2695-066 Bobadela LRS, Portugal
}




\section{Experimental}

The polycrystalline $\mathrm{LuZnSn}_{2}$ sample has been synthesized by reacting the elements at $\sim 1000^{\circ} \mathrm{C}$ followed by annealing at $400^{\circ} \mathrm{C}$, as described previously, and its crystal structure was determined from powder diffractogram using the FullProf program [23] for Rietveld refinements [1].

The bulk magnetic and specific heat measurements were performed in the temperature range of $1.9-300 \mathrm{~K}$ by means of the vibrating sample magnetometer (VSM) and the heat capacity (HC) options of the Quantum Design physical property measurement system (PPMS). The specific heat studies reported here were made in zero external magnetic field. Special care was taken to correct in a proper way the raw $\mathrm{HC}$ results taking into account the specific heat contribution originating from the adhesive addenda (Apiezon $N$ ) that is used to couple a given sample to the PPMS HC platform.

The ${ }^{119} \mathrm{Sn}$ Mössbauer spectrum has been measured at room temperature utilizing ${ }^{119 \mathrm{~m}} \mathrm{Sn}$ source in $\mathrm{CaSnO}_{3}$ matrix. A Mössbauer spectrometer of an electromechanical type was used in the constant-acceleration mode. The $23.875 \mathrm{keV} \gamma$-rays were detected with a proportional counter. The velocity scale was calibrated at room temperature with a ${ }^{57} \mathrm{Co}(\mathrm{Rh})$ source and a metallic iron foil. The obtained resonance absorption spectrum of ${ }^{119 \mathrm{~m}} \mathrm{Sn}$ was analyzed by means of least-squares fitting procedures using a full hyperfine interaction Hamiltonian within the transmission integral.

\section{Results and discussion}

\section{$X$ ray diffraction}

X-ray diffraction analysis showed that the $\mathrm{LuZnSn}_{2}$ compound crystallizes in the tetragonal $\mathrm{HfCuSi}_{2}$ structure type (P4/ $\mathrm{nmm}$ space group). Two symmetry inequivalent $\mathrm{Sn}$ sites $2 a(\overline{4} \mathrm{~m} 2)$ and $2 c(4 \mathrm{~mm})$, exist in the unit cell and a 1:1 nominal occupational ratio was observed [1].

\section{Heat capacity}

Figure 1 presents the temperature dependence of the specific heat. It can be seen that the total specific heat data $\left(C_{p}\right)$ can be fitted very well in the whole temperature range with the formula that accounts for the phonon and electronic contributions $\left(C_{p}=\right.$ $\left.C_{\text {lattice }}=C_{\mathrm{ph}}+C_{\mathrm{el}} ; C_{\mathrm{el}}=\gamma T\right)$ :

$$
\begin{aligned}
C_{p} & =C_{\mathrm{ph}+\mathrm{el}}=9 R \frac{1}{1-\alpha T}\left(\frac{T}{\theta_{\mathrm{D}}}\right)^{3} \int_{0}^{\frac{\theta_{\mathrm{D}}}{T}} \frac{x^{4} e^{x}}{\left(e^{x}-1\right)^{2}} d x \\
& +R \frac{1}{1-\alpha T} \sum_{i} m_{i} \frac{\left(\frac{\theta_{\mathrm{E}_{i}}}{T}\right)^{2} e^{\theta_{\mathrm{E}_{i}} / T}}{\left(e^{\theta_{\mathrm{E}_{i}} / T}-1\right)^{2}}+\gamma T
\end{aligned}
$$

where $\theta_{\mathrm{D}}$ is the Debye temperature, $\theta_{\mathrm{E} i}(i=1,2$ or 3$)$ are the Einstein temperatures, $\alpha$ is the anharmonic coefficient, $R$ is the gas constant and $\gamma$ stands for an electronic specific heat coefficient. All parameters derived from the fit are gathered in Table 1 together with the applied multiplicities $m_{i}$ for each individual Einstein branch $\mathrm{E}_{i}$. The last column of Table 1 contains the parameters obtained previously using the standard Debye formula for the description of the pure phonon contribution [22]. It is clearly seen that these last parameters, in spite of simplifications that were used (see Ref. [22]) are in good agreement with those obtained in this work, pointing to a conclusion that the simple Debye formula gives rather good estimation for the Debye temperature $\theta_{\mathrm{D}}$, at least as a first approximation. It has to be also underlined, that the obtained value of $\gamma(\gamma=(5.28$ $\left.\pm 0.05) \mathrm{mJ} \cdot \mathrm{mol}^{-1} \cdot \mathrm{K}^{-2}\right)$ is typical for nonmagnetic metallic ternary compounds.

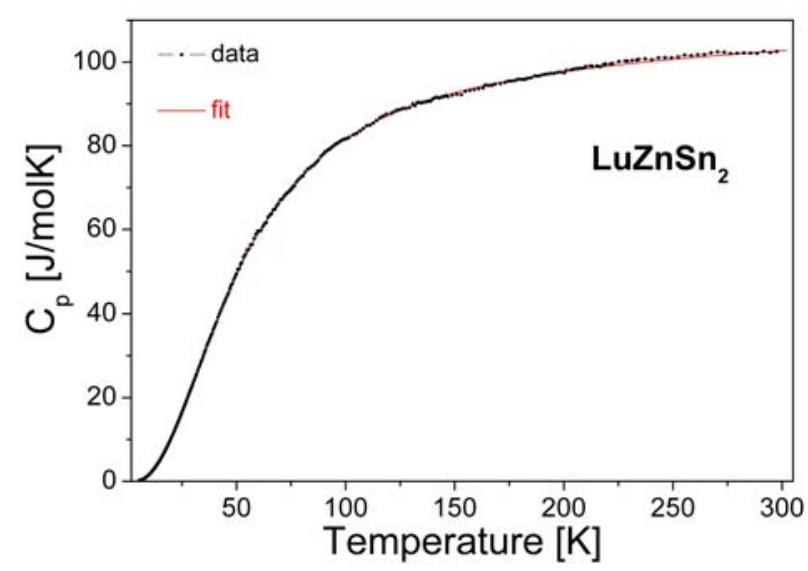

Fig. 1. Temperature dependence of the specific heat $\left(C_{p}\right)$ for the $\mathrm{LuZnSn}_{2}$. The continuous line presents the fit to $\mathrm{LuZnSn}_{2}$ data using the modified Debye expression (see Eq. (1)), as described in the text. The derived fitting parameters are gathered in Table 1.

Table 1. The results for the derived fitting parameters from heat capacity data using Eq. (1)

\begin{tabular}{|c|c|c|c|}
\hline$\overline{\theta_{\mathrm{D}}}$ & $(141.78 \pm 0.34) \mathrm{K}$ & & $(148.0 \pm 3.0) \mathrm{K}$ \\
\hline$\theta_{\mathrm{E}_{1}}$ & $(56.38 \pm 0.11) \mathrm{K}$ & $\times 1$ & \\
\hline$\theta_{\mathrm{E}_{2}}$ & $(156.50 \pm 0.47) \mathrm{K}$ & $\times 5$ & \\
\hline$\theta_{\mathrm{E}_{3}}$ & $(247.4 \pm 2.1) \mathrm{K}$ & $\times 3$ & \\
\hline$\alpha$ & $(13.08 \pm 0.77) \times 10^{-5} \mathrm{~K}^{-1}$ & & \\
\hline$\gamma$ & $(5.28 \pm 0.05) \times 10^{-3} \mathrm{~J} \cdot \mathrm{mol}^{-1} \cdot \mathrm{K}^{-2}$ & & $(5.75 \pm 0.20) \times 10^{-3} \mathrm{~J} \cdot \mathrm{mol}^{-1} \cdot \mathrm{K}^{-2}$ \\
\hline
\end{tabular}

$\begin{array}{cc}\text { Parameter } & \text { Multiplicity } \\ \left(m_{i}\right) & \text { Value }\end{array}$

Parameter values previously obtained in Ref. [22] using simplified assumptions 


\section{${ }^{119}$ Sn Mössbauer spectroscopy}

The point symmetries of Sn environments i.e. $\overline{4} m 2$ (2a site) and $4 m m$ ( $2 c$ site), ensure non-zero, axially symmetric (with asymmetry parameter $\eta=0$ ) electric field gradient tensor EFG at these sites, and hence a non-zero electric quadrupole hyperfine interactions at the Sn positions. In accord with the two non-cubic environments of Sn atom in the crystal structure (i.e. $2 a$ and $2 c$ sites) the obtained room temperature spectrum can be fitted with two quadrupole split components including single line impurity in the form of metallic tin (with isomer shift $\delta_{\text {is }}$ kept constant and equal to $2.552(9) \mathrm{mm} / \mathrm{s}$ [24]), which is also well established from X-ray analysis and originating from the synthesis procedure as well as the known decomposition process of the probe with time. The problem is that the applied fitting procedures are not unique, which has been illustrated in Fig. 2 for only two extreme examples.

Generally, to get reliable results, from a given fitting procedure, some parameters, like half widths for source $\Gamma_{\mathrm{s}}$ and absorber $\Gamma_{\mathrm{a}}$, have to be constrained and kept constant, being equal to those derived from earlier experiments with reference to $\mathrm{BaSnO}_{3}$ absorber. In this case these values are as follows: $\Gamma_{\mathrm{s}}=0.37 \mathrm{~mm} / \mathrm{s}$ and $\Gamma_{\mathrm{a}}=0.33 \mathrm{~mm} / \mathrm{s}$. Additionally, only fits with comparable relative intensities for both quadrupole components were accepted to reflect the fact that the occupation of above-mentioned $\mathrm{Sn}$ sites are equal. It must be stressed, that fits with constrained amplitudes of main Sn contributions with their 1:1 ratio, do not change remarkably the qoodnes, $\chi^{2}$, of such a fit. Small differences between relative intensities can be always explained by possible differences in Debye-Waller $f$ factors for both symmetry sites of tin. This is the main reason why in Table 2 the derived Mössbauer results are presented for fits with unconstrained main Sn contributions. The subtraction of the sum of the derived intensities for both quadrupole components from 100\% gives the contribution of the Sn impurity being of about $6 \%$ for different fit tries (see Table 2). Closer inspection of the obtained results (Table 2) clearly shows that both fits have almost the same quality factors $\chi^{2}$, in spite of the fact they lead to different interpretations. In the first case (fit number 1 , left side of Fig. 2) one can see that two Sn locations have much different isomer shifts but almost comparable quadrupole splittings, in frame of the observed error limits, while in the second case (fit number 2, right side of Fig. 2) the opposite situation takes place, i.e. isomer shifts are in turn almost the same but quadrupole splittings are quite different. It seems that this second situation describes in a much better way the physical reality, since for two different symmetry positions the observed quadrupole splittings should be rather different. As a matter of fact, such situation is observed in the closely related defected $\mathrm{GdT}_{x} \mathrm{Sn}_{2}$ compounds [21], where Sn atoms also occupy two different symmetry positions and where Mössbauer spectra can be fitted in a proper way only with two different quadrupole splittings, but with very similar isomer shifts for both Sn sites. On the other hand

Table 2. Hyperfine interaction parameters inferred from the ${ }^{119} \mathrm{Sn}$ resonance spectrum obtained for the $\mathrm{LuZnSn}_{2}$ intermetallic compound at $293 \mathrm{~K}$ using two different fitting procedures

\begin{tabular}{lccccc}
\hline Fit number & Component & $\begin{array}{c}\delta_{\text {is }} \\
{[\mathrm{mm} / \mathrm{s}]}\end{array}$ & $\begin{array}{c}\left|\Delta E_{Q}\right|^{*} \\
{[\mathrm{~mm} / \mathrm{s}]}\end{array}$ & $\begin{array}{c}\text { Relative area } \\
{[\%]}\end{array}$ & $\chi^{2}$ \\
\hline 1 & I & $2.000(7)$ & $2.236(12)$ & $44.5(1.4)$ & 0.9670 \\
2 & II & $2.383(6)$ & $2.224(15)$ & $49.0(1.3)$ & 0.9671 \\
\hline
\end{tabular}

${ }^{n} \Delta E_{Q}=e Q V_{z z}=2 \Delta$ where $\Delta$ is overall quadrupole splitting for a given component in the recorded spectrum (see Fig. 2).
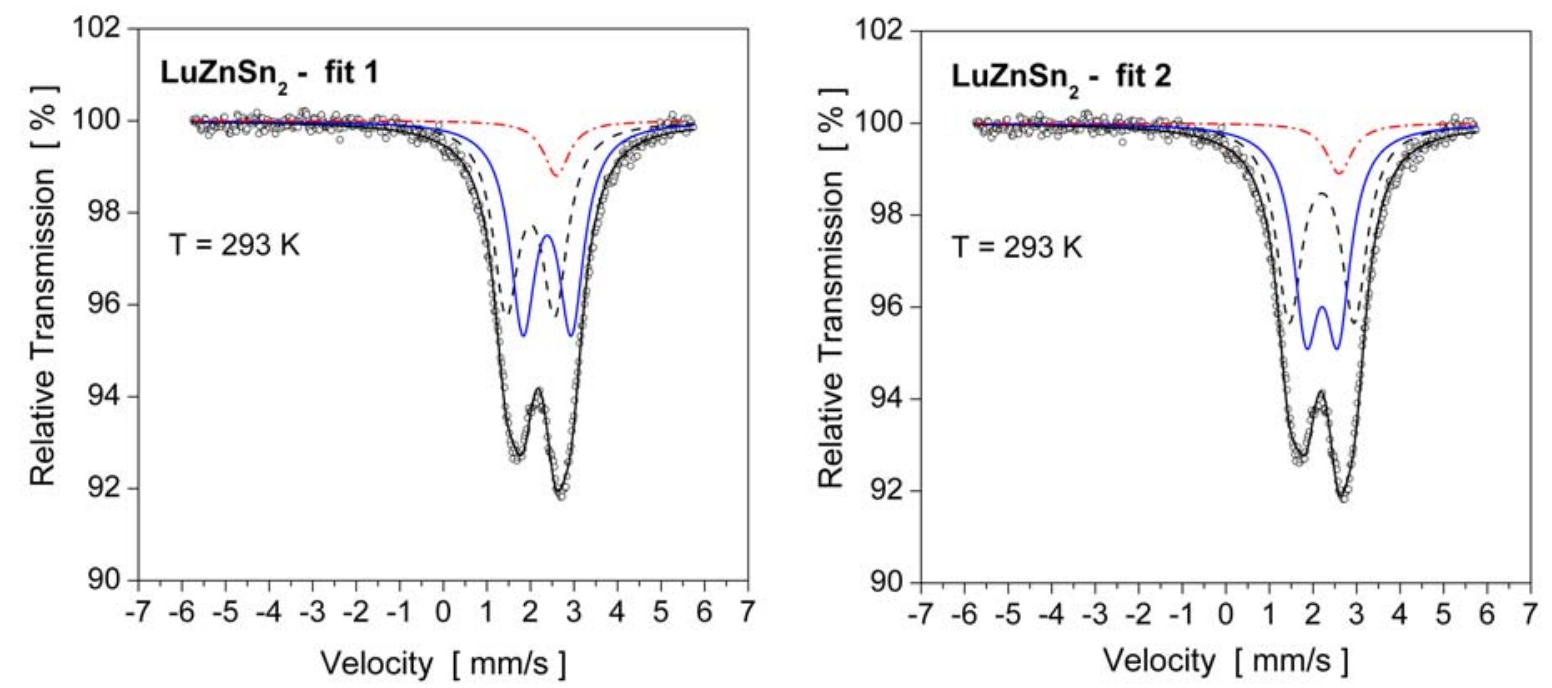

Fig. 2. ${ }^{119} \mathrm{Sn}$ Mössbauer spectrum of $\mathrm{LuZnSn}_{2}$ at room temperature fitted with two extreme fitting philosophies, using two quadrupole contributions and one strictly settled single line metallic tin impurity component (see text for explanation). 
an assumption that isomer shifts should be much different at Sn sites seems to be also unreasonable, since for Sn atoms their change requires a rather big change in valence electron $s p$ configuration of own tin atom [25], and this is not expected here, as in the $2 a$ position $\mathrm{Sn}$ atoms interact directly with other four Sn atoms and four Lu atoms, while at $2 c$ site interact with four $\mathrm{Zn}$ (instead of Sn) and four $\mathrm{Lu}$ atoms but valence $s$ configurations of $\mathrm{Sn}$ and $\mathrm{Zn}$ atoms stay roughly the same. The observed systematics of isomer shifts of $\mathrm{Sn}$ in most intermetallic systems (including ternary systems) [25] is in line, with the previous statement, showing that the effective number of $5 s$ tin electrons is merely the same and not much different than one (see Table 9a in Ref. [25]). In this context, one can state that a support from theory in predicting Mössbauer parameters would be of great importance. In particular, the theoretical calculations could enable the right association of the obtained sets of experimental results for hyperfine parameters to a given site in the crystallographic structure and also allowing for right interpretation of the derived isomer shifts and quadrupole splittings, giving a deeper insight into the electronic structure of the studied material. In this aspect one can only speculate, for example, that a larger quadrupole splitting could be associated to lower symmetry site of Sn. Nowadays, the use of density functional theory (DFT), is quite successful in this matter, but up to our best knowledge such calculations were not made for Sn hyperfine parameters in the system under study.

The values of the electric field gradient $V_{z z}$ at the tin nuclei can be obtained using the quadrupole interaction constants $\Delta E_{Q}=e Q V_{z z}$ (where nuclear quadrupole moment $Q$ for the first excited state of ${ }^{119 \mathrm{~m}} \mathrm{Sn}$ is equal to $Q=-0.094 \mathrm{~b}$ [26]) given in Table 2 by the simple relation: $V_{z z}=-8.47128 \times 10^{21}$ $\Delta E_{\mathrm{Q}}[\mathrm{mm} / \mathrm{s}] \mathrm{V} / \mathrm{m}^{2}$ and for the preferred fit number 2 they are $\left|V_{z z 1}\right|=25.646 \times 10^{21} \mathrm{~V} / \mathrm{m}^{2}$ and $\left|V_{z z 2}\right|$ $=12.664 \times 10^{21} \mathrm{~V} / \mathrm{m}^{2}$, respectively.

\section{Conclusions}

It was shown here that the experimental heat capacity data, $C_{p}(T)$, for $\mathrm{LuZnSn}_{2}$ can be successfully fitted in the whole temperature range taking into account anharmonic effects occurring at elevated temperatures, as well as by inclusion of respectively chosen Einstein modes. In this way, the observed above $36 \mathrm{~K}$ fit deviations from the $C_{p}(T)$ experimental results when these data are analyzed in term of simplified Debye model [22] were canceled.

The resonance spectrum recorded at room temperature can be fitted with different pairs of main quadrupole split subsets of hyperfine parameters for two crystalographically inequivalent $\mathrm{Sn}$ sites in $\mathrm{LuZnSn}_{2}$. However, from a physical point of view, only fit with two different quadrupole interaction constants and comparable isomer shifts for both Sn sites is acceptable. Those obtained by ${ }^{119 m}$ Sn Mössbauer spectroscopy quadrupole interaction constants allow for the determination of $V_{z z}$ components of electric field gradient (EFG) tensor at both Sn sites in $\mathrm{LuZnSn}_{2}$. The derived isomer shifts of about $2.20 \mathrm{~mm} / \mathrm{s}$ are characteristic for tin in metallic systems.

Acknowledgments. The magnetic and heat capacity measurements were carried out with the PPMS - Quantum Design equipment as well as Mössbauer facility purchased thanks to the financial support of the European Regional Development Fund in the framework of the Polish Innovation Economy Operational Program (contract no. POIG.02.01.00-12-023/08).

\section{References}

1. Verbovytskyy, Yu., Łątka, K., Przewoźnik, J., Leal, N., \& Gonçalves, A. P. (2012). On the new ternary $\mathrm{RZnSn}_{2}$ compounds with $\mathrm{HfCuSi}{ }_{2}$ structure type. Intermetallics, 20, 176-182. DOI: 10.1016/j.intermet.2011.08.024.

2. Görlich, E. A., Kmieć, R., Łątka, K., Matlak, T., Ruebenbauer, K., Szytuła, A., \& Tomala, K. (1975). Transferred hyperfine fields at the tin site in the Heusler-type alloys $\mathrm{Co}_{2} \mathrm{YSn}(\mathrm{Y}=\mathrm{Ti}, \mathrm{Zr}, \mathrm{Hf}, \mathrm{V})$. Phys. Status Solidi A-Appl. Mat., 30, 765-770. DOI: 10.1002/pssa.2210300237.

3. Görlich, E. A., Kmieć, R., Łątka, K., Szytuła, A., \& Zygmunt, A. (1994). Magnetic properties and ${ }^{119} \mathrm{Sn}$ hyperfine interactions investigated in $\mathrm{RCoSn}(\mathrm{R}=\mathrm{Tb}$, Dy, Ho, Er) compounds. J. Phys.-Condens. Matter, 6, 11127-11139. DOI: 10.1088/0953-8984/6/50/020.

4. Troć, R., Tran, V. H., Kolenda, M., Kruk, R., Łątka, K., Szytuła, A., Rossat-Mignod, J., Bonnet, M., \& Büchner, B. (1995). X-ray and neutron diffraction studies of UPdSn. J. Magn. Magn. Mater., 151, 102-110. DOI: 10.1016/0304-8853(95)00396-7.

5. Kruk, R., Kmieć, R., Łątka, K., Tomala, K., Troć, R., \& Tran, V. H. (1996). ${ }^{119}$ Sn Mössbauer studies of the compound UAuSn. J. Alloy. Compd., 232, L8-L11. DOI: 10.1016/0925-8388(95)02008-X.

6. Kruk, R., Kmieć, R., Łątka K., Tomala, K., Troć, R., \& Tran, V. H. (1997). Magnetic properties of UTSn compounds ( $\mathrm{T}=\mathrm{Co}, \mathrm{Rh}$, Ir, Ru) studied by ${ }^{119} \mathrm{Sn}$ Mössbauer spectroscopy. Phys. Rev. B, 55, 5851-5857. DOI: 10.1103/PhysRevB.55.5851.

7. Łątka, K., Görlich, E. A., Chajec, W., Kmieć, R., \& Pacyna, A. W. J. (1997). Crystal and magnetic properties of GdTSn compounds $(\mathrm{T}=\mathrm{Ag}, \mathrm{Au})$. J. Alloy. Compd., 262/263, 108-113.

8. Łątka, K., Görlich, E. A., Kmieć, R., Kruk, R., Pacyna, A. W. J., \& Chajec, W. (1998). Looking at unusual magnetism in rare-earth intermetallics with ${ }^{119} \mathrm{Sn}$ Mössbauer spectroscopy. Mol. Phys. Rep., 22, 87-97.

9. Görlich, E. A., Łątka, K., Kmieć, R., \& Warkocki, W. (1998). Hyperfine interactions of ${ }^{155} \mathrm{Gd}$ in the spin-glass systems GdAgSn and GdAuSn. Mol. Phys. Rep., 22, 35-40.

10. Łątka, K., Kmieć, R., \& Gurgul, J. (2001). ${ }^{119}$ Sn Mössbauer spectroscopy studies of RAgSn compounds (R=La, Ce, Pr). J. Alloy. Compd., 319, 43-49. DOI: 10.1016/S0925-8388(01)00896-9.

11. Łątka, K., Chajec, W., Kmieć, R., \& Pacyna, A. W. J. (2001). Magnetic susceptibility and ${ }^{119}$ Sn Mössbauer spectroscopy studies of RAuSn compounds $(\mathrm{R}=\mathrm{La}$, Ce, Pr). J. Magn. Magn. Mater., 224, 241-248. DOI: 10.1016/S0304-8853(01)00035-X.

12. Łątka, K., Kmieć, R., Kruk, R., Pacyna, A. W., Rams, M., Schmidt, T., \& Pöttgen, R. (2003). Exotic phase transitions in RERhSn compounds. Nukleonika, 48(Suppl. 1), 35-40. 
13. Łątka, K., Kmieć, R., Kruk, R., Pacyna, A. W., Rams, M., Schmidt, T., Kotzyba, G., Pöttgen, R., \& Johrendt, D. (2003). Structure and properties of CeRhSn - a valence fluctuating system. Acta Phys. Pol. B, 34(2), $1225-1229$.

14. Łątka, K., Kmieć, R., Gurgul, J., Rams, M., Pacyna, A. W., Schmidt, T., \& Pöttgen, R. (2005). Structure, magnetic properties and ${ }^{119} \mathrm{Sn}$ Mössbauer spectroscopy of PrRhSn. J. Solid State Chem., 178, 3101-3109. DOI: 10.1016/j.jssc.2005.06.041.

15. Schmidt, T., Johrendt, D., Sebastian, C. P., Pöttgen, R., Łątka, K., \& Kmieć, R. (2005). Structure, chemical bonding, and ${ }^{119} \mathrm{Sn}$ Mössbauer spectroscopy of LaRhSn and CeRhSn. Z. Naturforsch., 60b, 1036-1042.

16. Łątka, K., Kmieć, R., Kruk, R., Pacyna, A. W., Fickenscher, T., Hoffmann, R. -D., \& Pöttgen, R. (2005). Structure, magnetic properties and Mössbauer spectroscopy of GdRhSn. J. Solid State Chem., 178, 2077-2090. DOI: 10.1016/j.jssc.2005.04.010.

17. Łątka, K., Kmieć, R., Gurgul, J., Pacyna, A. W., Rams, M., Schmidt, T., \& Pöttgen, R. (2006). Magnetic ordering in NdRhSn. J. Magn. Magn. Mater., 301, 359-370. DOI: 10.1016/j.jmmm.2005.07.009.

18. Łątka, K., Kmieć, R., Kruk, R., Pacyna, A. W., Rams, M., Schmidt, T., \& Pöttgen, R. (2008). Electronic and magnetic properties of ternary stannides RERhSn $(\mathrm{RE}=$ light rare-earth metals). J. Magn. Magn. Mater., 320(3/4), L18-L20. DOI: 10.1016/j.jmmm.2007.05.031.

19. Łątka, K., Pacyna, W., Pöttgen, R., \& Schappacher, F. M. (2008). Puzzling magnetism of $\mathrm{Gd}_{3} \mathrm{Cu}_{4} \mathrm{Sn}_{4}$. Acta Phys. Pol. A, 114, 1501-1508.
20. Łątka, K., Kruk, R., Kmieć, R., Troć, R., \& Tran, V. H. (2010). ${ }^{119}$ Sn Mössbauer spectroscopy in the heavy-fermion ferrimagnet $\mathrm{UCu}_{5} \mathrm{Sn}$ ". J. Phys.-Conf. Series, 217, 012136(1-4). DOI: 10.1088/17426596/217/1/012136.

21. Görlich, E. A., Łatka, K., Kmieć, R., Tomkowicz, Z., \& Warkocki, W. (2000). Hyperfine interactions of ${ }^{119} \mathrm{Sn}$ and ${ }^{155} \mathrm{Gd}$ versus bulk properties of $\mathrm{GdT}_{\mathrm{x}} \mathrm{Sn}_{2}$ phases. Mol. Phys. Rep., 30, 59-65.

22. Łątka, K., Przewoźnik, J., Verbovytskyy, Yu., \& Gonçalves, A. P. (2013). Thermodynamic properties of $\mathrm{RZnSn}_{2}(\mathrm{R}=\mathrm{Y}, \mathrm{Er}, \mathrm{Lu})$ compounds with $\mathrm{HfCuSi}_{2}$ structure type. Solid State Phenomena, 194, 67-70. DOI: 10.4028/www.scientific.net/SSP.194.67.

23. Rodriguez-Carvajal, J., \& Roisnel, T. (1998). FullProf.98 and WinPLOTR: New Windows 95/NT Applications for Diffraction Commission for Powder Diffraction, International Union for Crystallography, Newsletter no. 20 (May-August) Summer.

24. Stevens, J. G., \& Gettys, W. I. (1978). Mössbauer isomer shift reference scales. In G. K. Shenoy \& F. E. Wagner (Eds.), Mössbauer isomer shifts (pp. 901-906). Amsterdam: North-Holland Publishing Co.

25. Flinn, P. (1978). Tin isomer shifts. In G. K. Shenoy \& F. E. Wagner (Eds.), Mössbauer isomer shifts (pp. 593-616). Amsterdam: North-Holland Publishing Co.

26. Mössbauer Effect Data Center (MEDC). (2010, July). Properties of isotopes relevant to Mössbauer spectroscopy. ${ }^{119} \mathrm{Sn}$ isotope properties. Retrieved June 3, 2014, from http://www.medc.dicp.ac.cn/Resourcesisotopes/Resource-Sn.php. 